\title{
Broadband Homonuclear Decoupling in Heteronuclear Shift Correlation NMR Spectroscopy
}

\author{
AD BAX \\ Department of Chemistry, Colorado State University, Fort Collins, Colorado 80523
}

Received March 7, 1983

Recently, Garbow, Weitekamp, and Pines introduced a new approach to homonuclear broadband decoupling (1). Independently, a closely related experiment has been proposed for semiselective heteronuclear $J$ spectroscopy (2). The two types of experiments have the common feature that all protons which are not directly attached to a ${ }^{13} \mathrm{C}$ nucleus experience an effect as if a nonselective $180^{\circ}$ pulse were applied, while protons bonded to a ${ }^{13} \mathrm{C}$ nucleus are not affected. This type of behavior can be used conveniently in many of the heteronuclear types of two-dimensional experiments (3-9). Here, its use for sensitivity and resolution enhancement in heteronuclear spectroscopy will be demonstrated. It is mentioned here that other approaches to homonuclear broadband decoupling in heteronuclear shift correlation spectroscopy have been proposed by Frenkiel (10), but those experiments rely on a different principle (1I), and are of a less general nature.

The experimental scheme is sketched in Fig. 1. The theory of the conventional heteronuclear decoupled shift correlation experiment (3) has been described elsewhere (3-6) and will be repeated here. The difference between the conventional heteronuclear decoupled shift correlation experiment and the scheme of Fig. 1 is that the single $180^{\circ}{ }^{13} \mathrm{C}$ pulse at the center of the evolution period has now been replaced by a $90^{\circ}{ }_{x}\left({ }^{1} \mathrm{H}\right)-1 /(2 J)-180^{\circ}{ }_{x}\left({ }^{1} \mathrm{H}\right), 180^{\circ}{ }_{x}\left({ }^{13} \mathrm{C}\right)-1 /(2 J)-90^{\circ}{ }_{-x}\left({ }^{1} \mathrm{H}\right)$ sequence, where $J$ is the magnitude of the heteronuclear coupling constant in hertz. It has been shown $(1,2)$ that this sequence has the effect of a single $180^{\circ}$ pulse for those protons that are not attached to a ${ }^{13} \mathrm{C}$ nucleus, while other protons (those attached to ${ }^{13} \mathrm{C}$ ) are essentially

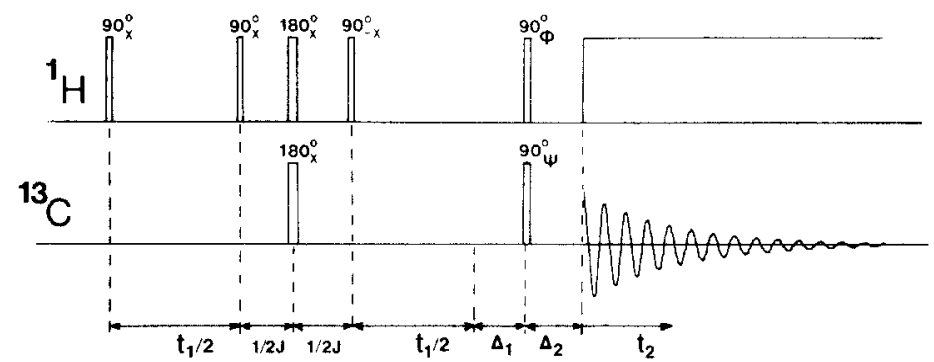

FIG. 1. Pulse scheme of the homonuclear broadband decoupled shift correlation experiment. The delays $\Delta_{1}$ and $\Delta_{2}$ are set to the same values as in the conventional shift correlation experiment $(3,6)$. The phases $\phi$ and $\psi$ are cycled according to Table 1 . 
unaffected. If a proton, $\mathrm{A}$, directly coupled to a ${ }^{13} \mathrm{C}, \mathrm{X}$, is also coupled to a proton, $\mathrm{M}$, which is not attached to ${ }^{13} \mathrm{C}$ and is initially, for example in the $m=1 / 2$ spin state, the pulse sequence inverts the state of $M$ to $m=-1 / 2$, and does not affect the (transverse) A magnetization. If the sequence is applied at the center of the evolution period, there will be no overall effect of coupling between $A$ and $M$ at the end of the evolution period, because the A nucleus is coupled for a time $t_{1} / 2$ to the $\mathrm{M}$ nucleus in the $m=1 / 2$ state and for another time $t_{1} / 2$ to the $\mathrm{M}$ nucleus in the $m=-1 / 2$ state. Therefore, the position of the A magnetization vector in the transverse plane depends only on the chemical shift, $\delta_{\mathrm{A}}$, and the length of the evolution period $t_{1}$. In the usual way (3-5), this magnetization of proton $\mathrm{A}$ is then transferred to its directly coupled ${ }^{13} \mathrm{C}$ nucleus, $\mathrm{X}$. Therefore, the observed ${ }^{13} \mathrm{C}$ signal will be modulated by the chemical shift, $\delta_{\mathrm{A}}$, only. If broadband heteronuclear proton decoupling is employed during

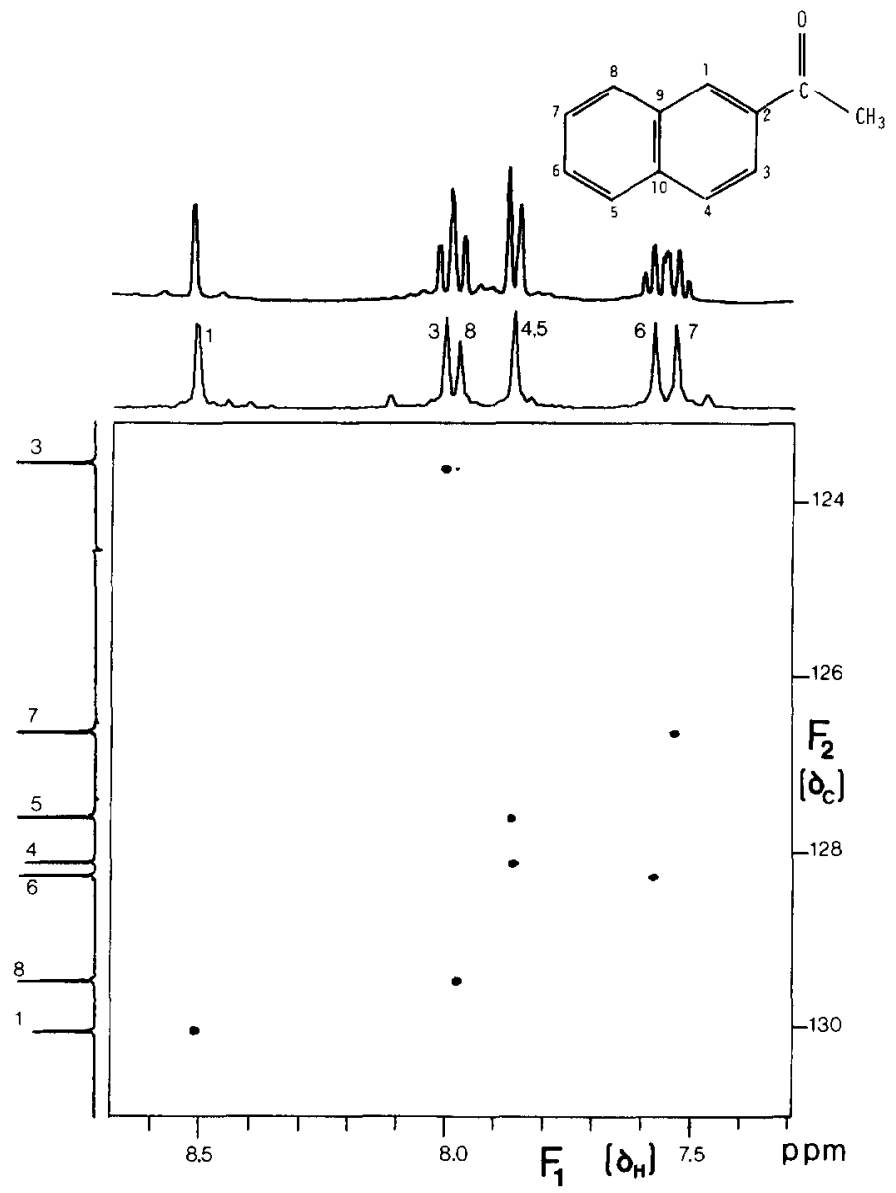

FIG. 2. Homonuclear broadband decoupled absolute-value-mode heteronuclear shift correlation spectrum of the aromatic resonances in 2-acetonaphtalene (inset). A $192 \times 512$ data matrix was acquired, and four experiments were performed for each value of $t_{1}$, with the phases of the if pulses as given in Table 1. Along the $F_{2}$ axis, the corresponding conventional ${ }^{13} \mathrm{C}$ spectrum is shown. Along the $F_{1}$ axis, the projection of the absolute value mode $2 \mathrm{D}$ spectrum on this axis, and the conventional proton spectrum are shown. 
acquisition $\left(t_{2}\right)$, a two-dimensional Fourier transformation will give a single resonance at $\left(F_{1}, F_{2}\right)=\left(\delta_{\mathrm{A}}, \delta_{\mathrm{X}}\right)$. No homonuclear proton splittings will appear in the $F_{1}$ dimension.

Experiments were performed on a NT-360 spectrometer, controlled by a 1180 computer and a $293 \mathrm{~A}^{\prime}$ pulse programmer. A $1 M$ concentration of 2-acetonaphtalene in acetone- $d_{6}$ in a $12 \mathrm{~mm}$ sample tube was used. Figure 2 shows a proton-proton decoupled 2D shift correlation spectrum of the protonated aromatic region. A 192 $\times 512$ data matrix was acquired and four experiments were performed for each value of $t_{1}$. The total measuring time was approximately $40 \mathrm{~min}$. The phases of the final proton and ${ }^{13} \mathrm{C}$ pulses in the scheme of Fig. 1 were cycled in the four steps to allow distinguishing between positive and negative proton modulation frequencies and for suppression of axial peaks at $F_{1}-0 \mathrm{~Hz}(5,6)$. The cycling of the if pulses actually employed here is given in Table 1 , and all data are co-added with identical receiver phase (the only option with the NT-360 used). Along the $F_{2}$ axis, the conventional ${ }^{1} \mathrm{H}$-decoupled ${ }^{13} \mathrm{C}$ spectrum is shown. Along the $F_{1}$ axis, an absolute value mode projection of the $2 \mathrm{D}$ spectrum on this axis is shown, representing the homonuclear broadband decoupled proton spectrum. For comparison, the conventional proton spectrum, recorded on the same sample in the same $12 \mathrm{~mm}$ sample tube, is also shown along this axis. Absorption mode cross sections parallel to the $F_{1}$ axis through the data matrix of Fig. 2 taken at the various ${ }^{13} \mathrm{C} F_{2}$ frequencies are shown in Fig. 3. Those cross sections demonstrate the excellent proton resolution that can be obtained. In this case, the main limitation for high resolution in the $F_{1}$ dimension is the limited acquisition time in this dimension $(384 \mathrm{msec})$. Gaussian multiplication was used in both dimensions to avoid truncation. Small mirror images with respect to the proton transmitter frequency (midpoint of the traces) can be seen in Fig. 3; these images are attributable to imperfect $90^{\circ}$ phase shifts in the decoupler channel.

In cases where high $F_{1}$ resolution is required and ${ }^{1} \mathrm{H}-{ }^{1} \mathrm{H}$ splittings in the conventional experiment would be resolved, the sensitivity of the new, homonuclear decoupled version should be better, because the sensitivity is proportional to the reciprocal of the number of resonances over which signal energy is being distributed (12). On the other hand, one loses information about the proton-proton couplings, which sometimes could be of practical use if the proton spectrum shows severe overlap. It has been found cxpcrimentally (6), that in selecting the delays $1 /(2 J)$ at the center of the

TABLE 1

The if Phases of the final Proton

PULSE $\phi$ AND OF THE FINAL ${ }^{13} \mathrm{C}$

PULSE $\psi$ IN THE FOUR STEPS OF THE EXPERIMENT OF FIG. $1^{a}$

\begin{tabular}{rr}
\hline$\phi$ & $\psi$ \\
$x$ & $x$ \\
$y$ & $-y$ \\
$-x$ & $-x$ \\
$-y$ & $y$ \\
\hline
\end{tabular}

a All data are co-added with identical receiver phase. 


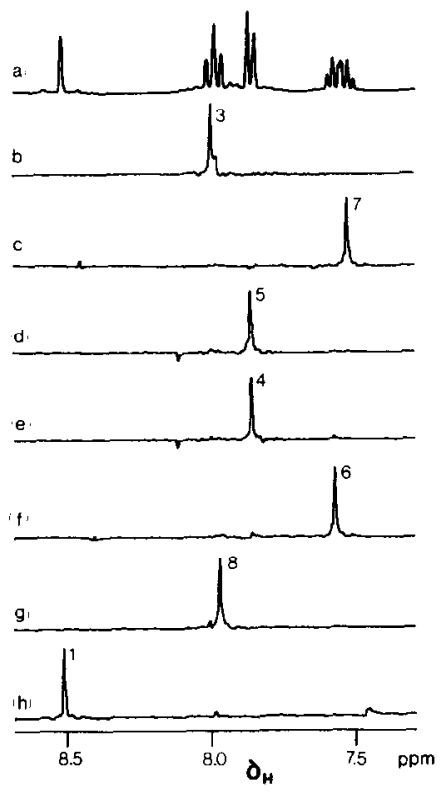

Fig. 3. (a) The conventional proton spectrum of the aromatic resonances in 2-acetonaphtalene, and (bh) absorption mode cross sections parallel to the $F_{1}$ axis through the data matrix of Fig. 2 for carbons $\mathrm{Cl}$, $\mathrm{C} 8, \mathrm{C} 6, \mathrm{C} 4, \mathrm{C} 5, \mathrm{C} 7$, and $\mathrm{C} 3$, respectively.

evolution period, the value of $J$ for the direct heteronuclear coupling has to be accurate to within approximately $10 \%$. It also has been found that the homonuclear proton multiplet width has to be a factor of five or more smaller than the value of $J$. Garbow et al. ( $I$ ) reported that other sequences for selectively inverting spin states of protons that are not bonded to a ${ }^{13} \mathrm{C}$ are less sensitive to the limitations mentioned above, and their applicability in heteronuclear spectroscopy is under investigation.

\section{ACKNOWLEDGMENT}

The author is indebted to Professor G. E. Maciel for many valuable comments in preparing the manuscript and further gratefully acknowledges partial support of this work from the U.S. Department of Energy (Laramie Energy Technical Center) and use of the Colorado State University Regional NMR Center, supported by National Science Foundation Grant CHE-78-18581.

\section{REFERENCES}

1. J. R. Garbow, D. P. Weitekamp, and A. Pines, Chem. Phys. Lett. 93, 504 (1982).

2. A. BAX, J. Magn. Reson., in press.

3. A. A. Maudsley, L. Muller, AND R. R. ERnSt, J. Magn. Reson. 28, 463 (1977).

4. R. Freeman, Proc. Roy. Soc. London, Ser. A 373, 149 (1980).

5. A. BAX AND G. A. Morris, J. Magn. Reson. 42, 501 (1981).

6. A. BAX, "Topics in "'C NMR" (G. C. Levy, Ed.), Vol. 4, Wiley Interscience, in press.

7. P. H. Bolton and G. Bodenhausen, Chem. Phys. Lett. 89, 139 (1982).

8. R. FrEeman AND G. A. MorRIS, Bull. Magn. Reson. 1, 5 (1979).

9. L. Muller, J. Am. Chem. Soc. 101, 4481 (1979).

10. T. A. FrenkIEL, Part II thesis, Oxford University, 1980.

11. A. BAX, A. F. MehlKopf, AND J. SMidT, J. Magn. Reson. 35, 167 (1979).

12. W. P. Aue, P. BachmanN, A. Wokaun, AND R. R. ERnst, J. Magn. Reson. 29, 523 (1979). 